H I G H L I G H TS

PROTEIN SORTING

\section{Giga-sorting in the Golgi}

In the Golgi, mannose-6-phosphate receptors (M6PRs) grab newly synthesized lysosomal hydrolases by their sugars to whizz them to their final destination, lysosomes. But how do the receptors themselves know where to go? Two groups now report in Science that the GGA coat adaptors show them the way.

The lysosomal sorting signal was identified almost a decade ago - an acidic cluster followed by a dileucine motif in the cytoplasmic tail of the M6PR. The AP-1 adaptor complex was the prime suspect for binding M6PRs and assembling the clathrin coat. It now turns out that it is, in fact, the most recently discovered adaptors — the GGAs (for Golgi-localized, $\gamma$ ear-containing, ARF-binding) — that bind to the lysosomal targeting signals of M6PRs and sort them to lysosomes.

GGAs are made of four domains: a VHS domain; a GTP-ARF-binding domain (GAT); a hinge that contains binding sites for clathrin; and a GAE domain that interacts with $\gamma$-synergin and other regulators of coat assembly. The sorting signals of M6PRs interacted specifically with the VHS domains of GGAs in two-hybrid assays, in pulldown assays and by surface plasmon resonance spectroscopy. Specifically, the cation-independent M6PR bound all three human GGAs, whereas the

yeast $\mathrm{MSH} 2$ that occur in human MSH2 in hereditary nonpolyposis colorectal cancer. These mutations enhanced survival of a telomerasedefective strain to a similar extent as $\mathrm{MSH} 2$ deletion. Inactivating telomerase and mismatch repair in another yeast, Kluyveromyces lactis - with a pattern of telomere sequence degeneracy similar to that found in human cells - yielded similar results, indicating that these observations may indeed extrapolate to events occurring in mismatch repair-defective human cells.

These experiments raise many questions for cancer research. Do human telomeres undergo more recombination in the absence of cation-dependent M6PR preferred GGA1 over GGA3, and did not bind GGA2. Immunofluorescence studies showed that M6PRs and GGA1 colocalize in the trans-Golgi network (TGN) and in the cell periphery, and time-lapse imaging of live cells revealed that GGA1 associates with tubules and vesicles that bud from the TGN.

Kornfeld and colleagues show that there is a correlation between reduced in vitro binding of the cation-independent MPR to GGA2 and mis-sorting of lysosomal enzymes. Last, Bonifacino and colleagues provide evidence for a functional involvement of GGAs in sorting of M6PRs, as overexpression of a dominant-negative GGA1 construct blocks exit of the cation-dependent M6PR from the TGN.

So GGAs probably function as adaptors between M6PRs, ARF, clathrin and regulators of coat assembly. But do they recruit receptors into AP-1-clathrin vesicles or do they build clathrin-coated vesicles of their own?

Raluca Gagescu

\section{(0) References and links} ORIGINAL RESEARCH PAPERS Puertollano, $R$. et al. Sorting of mannose 6-phosphate receptors mediated by the GGAs. Science 292, 1712-1716 (2001) | Zhu, Y. et al. Binding of GGA2 to the lysosomal enzyme sorting motif of the mannose 6 phosphate receptor. Science 292, 1716-1718 (2001)

mismatch repair? Can mismatch repair-defective cancer cells get by without telomerase? What is the relative importance of mutation versus telomere maintenance in these cells? And can we develop therapeutics that reduce mismatch repair-defective cells back to the status of mere mortals?

Cath Brooksbank Editor, Nature Reviews Cancer

(2) References and links ORIGINAL RESEARCH PAPER Rizki, A. \& Lundblad, $V$. Defects in mismatch repair promote telomerase-independent proliferation. Nature 411, 713-716 (2001) FURTHER READING Kucherlapati, R. \& DePinho, R. A. Telomerase meets its mismatch.

Nature 411, 647-648 (2001)

WEB SITE Mismatch repair

\section{IN BRIEF}

\section{PROTEIN DEGRADATION}

Impairment of the ubiquitin-proteasome system by protein aggregation.

Bence, N. F. et al. Science 292, 1552-1555 (2001)

Protein aggregates are a prominent feature of many neurodegenerative diseases. But are these aggregates a cause or a consequence of neurotoxicity? The authors show here that overexpression of proteins that are prone to aggregation - either in pericentriolar aggresomes or in amyloid-like fibrils - inhibits the ubiquitin-proteasome system. As proteasomal degradation is essential for cell survival, inhibition of this cellular system by protein aggregation is highly pathogenic.

\section{TECHNIQUE}

Specific targeting of insect and vertebrate telomeres with pyrrole and imidazole polyamides.

Maeshima, K. et al. EMBO J. 20, 3218-3228 (2001)

The authors report new cell-permeant compounds - polyamides containing two, flexibly linked, hairpin DNA-binding moieties that specifically target telomeres. Fluorescent analogues of these compounds colocalize with the telomere-binding proteins TRF1 and TRF2. These drugs provide a convenient alternative to current cumbersome methods for estimating telomere length. Polyamides of this type could become useful tools for telomere research, and might even have potential as medicinal agents.

\section{PRIONS}

Sensitive detection of pathological prion protein by cyclic amplification of protein misfolding.

Saborio, G. P. et al. Nature 411, 810-813 (2001)

The infectious prion protein, $\mathrm{PrP}^{\mathrm{sc}}$, is thought to work by catalysing the conversion of the cellular glycoprotein $\mathrm{PrP}^{\mathrm{C}}$ into its infectious form. So far, although there is evidence that prions are present in body fluids, it has proved difficult to detect the infectious prion in patients before death. Here, Saborio et al. use ultrasound to free prions from plaques in vitro, which speeds up the conversion cycle. This might allow diagnosis of the presence of infectious prions in the bloodstream.

\section{SIGNAL TRANSDUCTION}

\section{G-protein signalling through tubby proteins}

\section{Santagata, S. et al. Science 292, 2041-2050 (2001)}

Loss of tubby function leads to obesity. In this study, tubby is shown to act downstream of G-protein-coupled-receptors to potentially regulate gene transcription. Tubby localizes to the plasma membrane by interacting, through its carboxy-terminal 'tubby' domain, with phosphatidylinositol-4,5-bisphosphate $\left(\operatorname{PtdIns}(4,5) \mathrm{P}_{2}\right)$. However, activation of $\mathrm{G} \alpha_{\mathrm{q}}$-coupled receptors leads to PtdIns $(4,5) \mathrm{P}_{2}$ hydrolysis - probably by increasing phospholipase $C \beta$ activity. Tubby then translocates to the nucleus, where it might influence genes involved in energy homeostasis. 